\title{
L'utilisation du bois au village Laténien des Pichelots (Les Alleuds, Maine-et-Loire)
}

Gérard Aubin, Jean-Philippe Bouvet, Axel Levillayer, Jean Siraudeau et Michel Gruet

\section{(2) OpenEdition \\ Journals}

Édition électronique

URL : http://journals.openedition.org/rao/315

DOI : 10.4000/rao.315

ISBN : 978-2-7535-1607-6

ISSN : 1775-3732

\section{Éditeur}

Presses universitaires de Rennes

\section{Édition imprimée}

Date de publication : 30 décembre 2007

Pagination : 111-120

ISBN : 978-2-7535-0574-2

ISSN : 0767-709X

\section{Référence électronique}

Gérard Aubin, Jean-Philippe Bouvet, Axel Levillayer, Jean Siraudeau et Michel Gruet, «L'utilisation du bois au village Laténien des Pichelots (Les Alleuds, Maine-et-Loire) », Revue archéologique de l'Ouest [En ligne], 24 | 2007, mis en ligne le 30 décembre 2009, consulté le 03 décembre 2020. URL : http:// journals.openedition.org/rao/315; DOI : https://doi.org/10.4000/rao.315 


\title{
L'utilisation du bois au village Laténien des Pichelots (Les Alleuds, Maine-et-Loire)
}

\author{
Michel Gruet $\dagger$ \\ Présenté par Gérard Aubin*, Jean-Philippe Bouvet**, Axel Levillayer*** \\ et Jean Siraudeau ${ }^{* * * *}$
}

Présentation - Résumé : Découvert fortuitement à l'occasion de l'exploitation d'une gravière, probablement entre 1957 et 1963 , le site des Pichelots (coordonnées Lambert : $\mathrm{x}=389,9$ - 390,1; y = 2261,4 - 2261,7 ; réf. Carte archéologique de la France «Patriarche »: 49 001 0008) fit, sous la direction de M. Gruet, l'objet de fouilles ponctuelles à partir de 1973 - les premières relations commencent en 1971 - puis, à partir de 1980, d'études approfondies à la suite des décapages préliminaires systématiques des terres superficielles par l'exploitant de la carrière, Bernard Loriou (fait chevalier des Arts et Lettres en 1983). La surface actuellement étudiée approche les cinq hectares au total - l'extension estimée du village gaulois est de sept hectares environ - pour une exploitation actuelle la de carrière de neuf hectares environ. Le site fut fouillé par M. Gruet de 1973 à 1993, aidé de B. Passini et J. Siraudeau. Deux autres interventions ponctuelles en 1997 (D. Prigent et J.-Y. Hunot; Service départemental d'Archéologie du Conseil général de Maine-et-Loire) et en 2002 (J.-Ph. Bouvet et Rolande Simon-Millot; Direction régionale des Affaires culturelles des Pays de la Loire) complèteront les observations de M. Gruet. Du vivant de l'auteur (décédé en 1998; cf. L’Helgouac'h, 1998; Siraudeau, 1998), le site, pour ce qui concerne la période du second âge du Fer, n’a fait l'objet que de quelques publications préliminaires (notamment Gruet et Passini 1985; 1986) et d'un article consacré aux monnaies gauloises (Aubin et al., 1982). Le site en général, ainsi que le puits 30 et des mobiliers (amphores, monnaies, fibules et céramiques, pesons de métier à tisser notamment) ont été presentés sous forme de notices (13 au total) dans le catalogue consacré à l'exposition Nos ancêtres les gaulois aux marges de l'Armorique (Santrot et Meuret [dir.], 1999).

Une publication globale du site, structure par structure, assemblage de mobilier par assemblage, est en préparation (sous la direction de G. Aubin, J.- Ph. Bouvet, A. Levillayer et J. Siraudeau). Le village des Pichelots a fait aussi l'objet (par les mêmes auteurs) d'une notice en 2005 dans le cadre du Projet collectif de Recherche sur les agglomérations secondaires gallo-romaines de Bretagne et des Pays de la Loire. J. Siraudeau en a déjà étudié la totalité des amphores et le mobilier céramique des Pichelots, totalement réétudié, est intégré dans la thèse en cours de A. Levillayer.

Le site, de par le mobilier seulement publié à ce jour - nombreux fragments d'amphores républicaines, céramiques largement tournées, fibules, monnayage gaulois - a été placé à La Tène finale. Mais la reconstitution en cours des assemblages de mobilier expose une problématique complexe quant à la datation du village et à son évolution chronologique, à savoir de La Tène $\mathrm{C}$ à la fin de La Tène $\mathrm{D}$, voire au delà.

Néanmoins, il nous a semblé important de publier, en hommage à M. Gruet, ce manuscrit daté de $1988^{1}$, proposé par son fils Yves et consacré aux observations très précises que le fouilleur avait pu effectuer, malgré des conditions a priori défavorables, sur les structures en bois et autres matières organiques développées par les habitants des Pichelots. Ces structures en bois consistent en restes de poteaux correspondant à une palissade établie dans un fossé qui devait entourer l'habitat et à des constructions plus ou moins adossées à celle-ci (mais dont les plans n'ont pu être restitués). Cependant, ce sont surtout les traces de cuvelages repérées dans bon nombre de puits (plus de 31 furent étudiés en 1988) qui ont révélé tout un savoir-faire technique à partir de l'étude des pièces de bois préservées. Il ne faut pas négliger non plus les indices liés au tissage, à la tonnellerie ou à la décoration du bâti, ni les outils en fer et les ferrures associés au travail du bois ainsi qu’à sa mise en œuvre.

Toutes ces observations et études de M. Gruet, issues de ce manuscrit, seront intégrées à la publication générale du site en les réactualisant et en les replaçant dans les problématiques récentes de la recherche sur les âges du Fer.

\footnotetext{
* Inspecteur général de l'Archéologie. (gerard.aubin@culture.gouv.fr)

** Conservateur du Patrimoine; chef du service des stratégies territoriales, DRAC des Pays-de-la-Loire, Nantes.

*** Doctorant; UMR 6566, université de Rennes 1 (campus de Beaulieu), CS 74205, 35042 Rennes cedex.

*** Archéologue, Angers.

1. Ce manuscrit a été présenté pour publication après la tenue du colloque de l'AFEAF à Guéret (1988), dont l'une des thématiques concernait les matériaux périssables ou organiques. N'ayant pas fait l'objet d'une communication, cette étude n'avait pu être intégrée aux actes du colloque.
} 


\begin{abstract}
Presentation - Abstract: Wood utilization in the La Tene village of Les Pichelots (Les Alleuds, Maine-et-Loire)
Uncovered by chance through gravel quarrying in 1957-1963, the site of Les Pichelots has been sporadically studied by M. Gruet until he directed excavations there between 1973 and 1993, helped by B. Passini and J. Siraudeau. Complementary interventions occured in 1997 (by D. Prigent and J.-Y. Hunot) and in 2002 (by J.-Ph. Bouvet and R. Simon-Millot). The total studied area is not farshort of 5 hectares, for a total extension of the village estimated to nearly 7 hectares. During the lifetime, of the author (who died in 1998; cf. L'Helgouach 1998; Siraudeau, 1998), the site has only been the subject of a few preliminary reports (mostly Gruet and Passini 1985; 1986), plus a paper dedicated to the gaulish coins (Aubin et al., 1982). More recently, it has been presented through by a series of 13 notices in the catalogue of an exhibition (Santrot and Meuret, eds. 1999). A complete publication is in hand (under the direction of G. Aubin, J.-Ph. Bouvet, A. Levillayer and J. Siraudeau).

From the assemblage yet published (mostly wheel-turned pottery, numerous hards of Republican amphorae, Gaulish coinage) the site has been dated as late La Tene, but the studies in progress suggest an evolution from La Tene $C$ to the end of La Tene D, even later. Nevertheless, it seemed to us important to publish now this posthumous paper, dated 1988 and submitted by his son Yves. It is devoted to the very precise observations M. Gruet was able to carry out, despite very unfavourable conditions, on the wooden structures and other organic materials utilised by the inhabitants of Les Pichelots. The excavated wooden structures consist of post remains which correspond to a palisaded enclosure and buildings (unfortunately of undetermined plan) set against it. Much more significant were the traces of timbering still visible in many of the 31 wells excavated, the pieces preserved revealing a sophisticated technical knowledge. Moreover the site revealed evidence of weaving, cooperage and building decoration, plus iron tools and hinges linked to wood working use.
\end{abstract}

Mots clés : La Tène finale, habitat, charpenterie, puits, cuvelage, outillage, textile, décoration du bâti.

Key words: Late La Tene, settlement, timber work, well timbering, tools, weaving, building decoration.

On peut considérer que l'habitat gaulois des Pichelots est un village de plaine, tellement le plateau dont il occupe la légère pente vers le sud est peu élevé au-dessus des thalwegs environnants. Il est situé sur la commune des Alleuds, à $20 \mathrm{~km}$ au sud-est d'Angers et à $4 \mathrm{~km}$ au sud de la petite ville de Brissac. Il est établi sur une épaisse couche de graviers estuariens d'âge Cénomanien. La pierre est inexistante sur place et n'a pas été utilisée pour les constructions, pas même pour les fondations. Les traces de bâtiments y sont pourtant nombreuses et le bois a été largement utilisé. Le champ de fouille se trouve, encore actuellement, à moins de $100 \mathrm{~m}$ de l'orée de l'antique forêt de Brissac et, bien qu'il soit en bordure d'une voie protohistorique, l'habitat occupait très probablement une clairière de la forêt.

Les graviers quartzeux acides et secs où nous fouillons des structures en creux rebouchées, silos, dépotoirs, puits, sont absolument impropres à la conservation des débris ligneux, à deux exceptions près : les bois carbonisés et les bois situés en dessous de la nappe phréatique au fond des puits. Néanmoins, même dans les portions sèches superficielles, l'existence de bois peut être reconnue par les images virtuelles qu'en donnent les moulages des pièces ligneuses disparues lorsqu'une matrice en garde l'empreinte : c'est tantôt de l'argile plus ou moins cuite, tantôt des sables et graviers agglomérés par des oxydes naturels, parfois de la rouille au contact des objets de fer.

\section{La palissade (fig. 1)}

Une palissade de bois devait cerner le village (fig. 1, $\mathrm{n}^{\circ} 1$ ). Son implantation apparemment circulaire et légèrement sinueuse se faisait dans un étroit fossé que nous avons suivi sur une longueur de $230 \mathrm{~m}$. C'est contre ce fossé, du côté interne de sa courbure, que se massaient les trous de poteaux des constructions. La section du fossé montre une forme en Y avec une saignée centrale plus profonde pour recevoir la base de la palissade (coupe $\mathrm{n}^{\circ} 4$ ). L'existence de celle-ci nous est prouvée parce que, par endroits, nous avons constaté que deux remplissages nettement différents par leur granulométrie, l'un d'origine externe, l'autre interne, se heurtaient sur la ligne médiane, en une verticale, sans se mélanger, traduisant l'existence d'un écran séparateur. Autre constatation : deux fouilles minutieuses portant chacune sur une longueur de fossé de $5,5 \mathrm{~m}$ en deux points distants de $180 \mathrm{~m}\left(\mathrm{n}^{\circ} 1\right.$, zones $\mathrm{A}$ et $\mathrm{B}$ ), nous ont montré des enfoncements cylindriques de 15 à $30 \mathrm{~cm}$ de diamètre, disposés en quinconce irrégulier, dans le fond du fossé, sur ses pentes et sur ses bords. Les plans détaillés ( ${ }^{\circ} 2$ et 3 ), qui rendent compte de cette disposition (en courbes de niveaux) suggèrent l'étaiement de la palissade par des poteaux; consolidation peutêtre secondaire $\left(\mathrm{n}^{\circ} 4\right)$.

Le contenu de ce fossé est archéologiquement stérile, ce qui montre son implantation initiale avant l'habitat prolongé (lequel n'eut pas manqué de lui livrer quelques tessons). Un puits avec du matériel de La Tène III, qui recoupe cette palissade, en indique le terminus chronologique. 


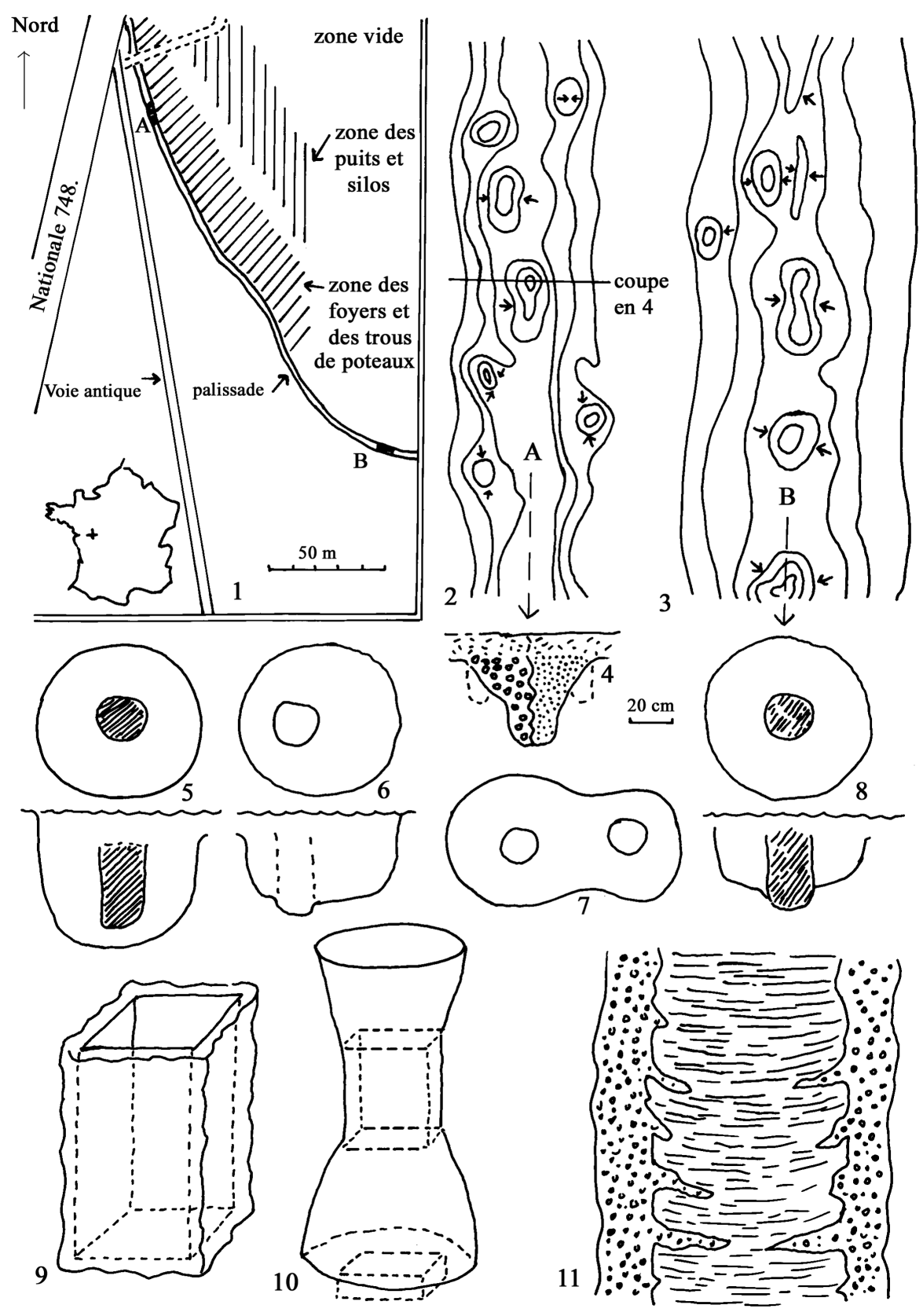

Figure $1: 1:$ carte de localisation du site (N-B : la " Nationale 748 " est aujourd'hui la RD 748) ; 2-3 : plan des deux secteurs fouillés du fossé palissadé ( $A$ et $B$ du plan 1); équidistance des courbes de niveau, $10 \mathrm{~cm}$ ) ; 4 : coupe du fossé (section A), au niveau du trou axial le plus profond, avec trous latéraux en projection; 5-8 : différents types de trous de poteaux (cf texte); 9 : structure initiale d'un puits (trou carré à cuvelage interne); 10 : état le plus fréquent à la découverte (seule la partie médiane et la base ont gardé leur section initiale); 11 : coupe d'un comblement de puits montrant les infiltrations de gravier entre les madriers aujourd'hui disparus.

Figure 1: 1 : location map and general plan of the site ( $N-B$ : "Nationale 748 " is today's $R D$ 748); 2-3 : plan of two sectors ( $A$ \& $B$ - see general plan 1) of the palisaded enclosure ditch (equidistance of contours: $10 \mathrm{~cm}$ ); 4 : section of ditch (part $A)$ with main axial post-hole and two lateral ones in projection; 5-8: different types of post-holes (see in text); 9: initial structure of a well (square hole with internal timbering); 10: general discovering condition (initial section preserved only in median part and waterlogged bottom); 11: section through the filling of a well, with gravel inflitrations between every (today disappeared) beams. 


\section{Les TROUS DE POTEAUX (fig. 1)}

Dans le gravier très serré qui constitue le sous-sol du gisement, il était impossible d'enfoncer un pieu par simple percussion; les habitants ont dû, ici comme ailleurs, creuser des fosses notablement plus grandes que le tronc auquel elles étaient destinées. De ces fosses, au plan généralement circulaire, nous n'avons que la partie inférieure puisqu'elles sont recouvertes de 30 à $50 \mathrm{~cm}$ de terre remaniée par les cultures actuelles (fig. 1, $\mathrm{n}^{\circ} 5$ ). Leur diamètre à l'ouverture varie de 35 à $120 \mathrm{~cm}$ avec une valeur médiane de $65 \mathrm{~cm}$. Dans le cas particulier et plus rare de fosses accueillant deux poteaux, elles peuvent être plus grandes et de forme allongée ou rectangulaire $\left(n^{\circ} 7\right)$.

Les trous de poteaux les plus indiscutables sont ceux qui nous montrent le fantôme noir du poteau lui-même. Seule la carbonisation, donc pratiquement l'incendie, a pu réaliser cette ombre formée d'un semis de braises ne dépassant pas le centimètre cube chacune, noyées dans une poudre de charbon de bois. Sur 263 trous 32 présentent ces fantômes. Ces taches ont régulièrement des sections circulaires mesurant 15 à $25 \mathrm{~cm}$ de diamètre. Sur une coupe verticale le cylindre sombre semble s'effiler vers le bas $\left(\mathrm{n}^{\circ} 5\right)$.

En l'absence de ces traces colorées, l'existence d'un poteau nous est souvent prouvée par la présence au fond du trou d'une cupule ou cuvette de faible diamètre légèrement surcreusée ( $\left.{ }^{\circ} 6\right)$. Étant donné la dureté du sol, il est peu probable qu'elle soit due au martèlement sur la tête du pieu. On peut croire qu'elle a été forée volontairement pour préciser plus exactement la position du pied du poteau. Il est fréquent, en effet, que ce surcreusement ne centre pas la grande cavité mais soit désaxé, rectifiant ainsi un alignement initial imprécis ( $n^{\circ}$ 6). Sur 263 trous de poteau, 41 montrent cette espèce d'ombilic, quelques-uns mêmes présentent à la fois fantôme et surcreusement $\left(n^{\circ} 8\right)$.

Malgré quelques alignements évidents, nos relevés ne permettent pas d'établir le plan des constructions dans l'enclos. La surabondance des trous est une gêne et le jeu des reconstitutions reste trop problématique. Il manque aussi tous les emplacements de pieux peu profondément enfoncés, maintenant dilués dans la terre arable remaniée. Outre le groupement déjà signalé contre la palissade d'enceinte, on note plus à l'intérieur, dans la zone des puits et des silos, des trous plus lâchement dispersés qui pouvaient soutenir une couverture légère au-dessus de ces structures (cf. plan général nº 1 ).

\section{Des PUITS BoIsÉs (fig. 1 et 2)}

Moins banal qu'une palissade ou des trous de poteaux dispersés, nous est apparu le cuvelage des puits des Pichelots. Dès le premier puits fouillé (locus 6) nous nous sommes rendu compte que, même lorsque la cavité que nous vidions était de forme cylindrique ou tronconique, la lumière du puits ancien devait avoir eu une section carrée. En effet, en avant des parois concaves de graviers en place plus ou moins excavées ou éboulées, de larges décollements par plans verticaux orthogonaux, parfois marqués par un feutrage de racines, séparaient des remplissages différents. Le comblement central, plus noir et souvent bourbeux, est plus riche en débris archéologiques alors que le comblement périphérique, plus clair et gravillonneux, reste presque ou tout à fait stérile. Par la suite tous les puits nous ont montré soit une coupe carrée sur toute leur hauteur $\left(n^{\circ} 9\right)$, ce qui est assez rare, soit au moins un segment présentant une telle section, segment généralement situé à moyenne profondeur et aussi tout au fond. Vers la surface, une fréquente forme en entonnoir semble due au ruissellement et, en profondeur, un peu au-dessus de la nappe phréatique, des affouillements réalisent des éboulements en cloche $\left(\mathrm{n}^{\circ} 10\right)$.

C'est aussi de la fouille du premier puits que nous avons déduit la présence d'un cuvelage de planches, en examinant les sections verticales. En effet, celles-ci montraient, régulièrement échelonnées en hauteur, des langues de graviers propres qui s'insinuaient dans la zone centrale plus sombre à partir de la zone périphérique $\left(n^{\circ} 11\right)$. On pouvait en conclure à des échappées, vers la lumière centrale du puits, de graviers provenant du remplissage périphérique et s'infiltrant entre des planches.

Finalement, le cinquième puits fouillé, en nous livrant un cuvelage de bois conservé en dessous de la nappe phréatique, nous a montré la justesse des précédentes déductions (cf. fig. 2). Nous avons d'abord extrait, à $6 \mathrm{~m}$ de profondeur et dans le plus grand désordre, 26 morceaux de bois fendus en long. Huit d'entre eux seulement dépassaient $75 \mathrm{~cm}$ de longueur; leurs extrémités étaient soit arrondies et comme polies $\left(n^{\circ} 16\right)$, soit au contraire déchiquetées et comme échevelées $\left(n^{\circ} 17\right)$. Plus bas, le cuvelage quadrangulaire était intact sur plus d'un mètre de haut. Il est formé de bastaings disposés en carré et réunis dans les angles par des assemblages de type mi-bois ( $\left.\mathrm{n}^{\circ} 12\right)$.

J'ai employé l'expression à mi-bois parce qu'elle est bien connue, mais en réalité ici les ajustages sont plus proches $\mathrm{du}$ tiers et c'est beaucoup mieux ainsi. En effet, dans un ajustage à mi-bois, ou bien on fait un montage inversé $\left(n^{\circ} 13\right)$ et les cadres superposés peuvent alors glisser les uns sur les autres sous une poussée latérale, ce qui est néfaste dans un puits, ou bien on fait un montage de même sens ( $\left.n^{\circ} 14\right)$ qui évite l'inconvénient précédent. Mais alors les tenons deviennent porteurs et si les mesures en hauteur des bastaings ne sont pas impeccablement égales on risque qu'ils ne soient plus jointifs. Dans l'assemblage que nous avons ici, proche du tiers avec un montage inversé, chaque bastaing repose direc- 


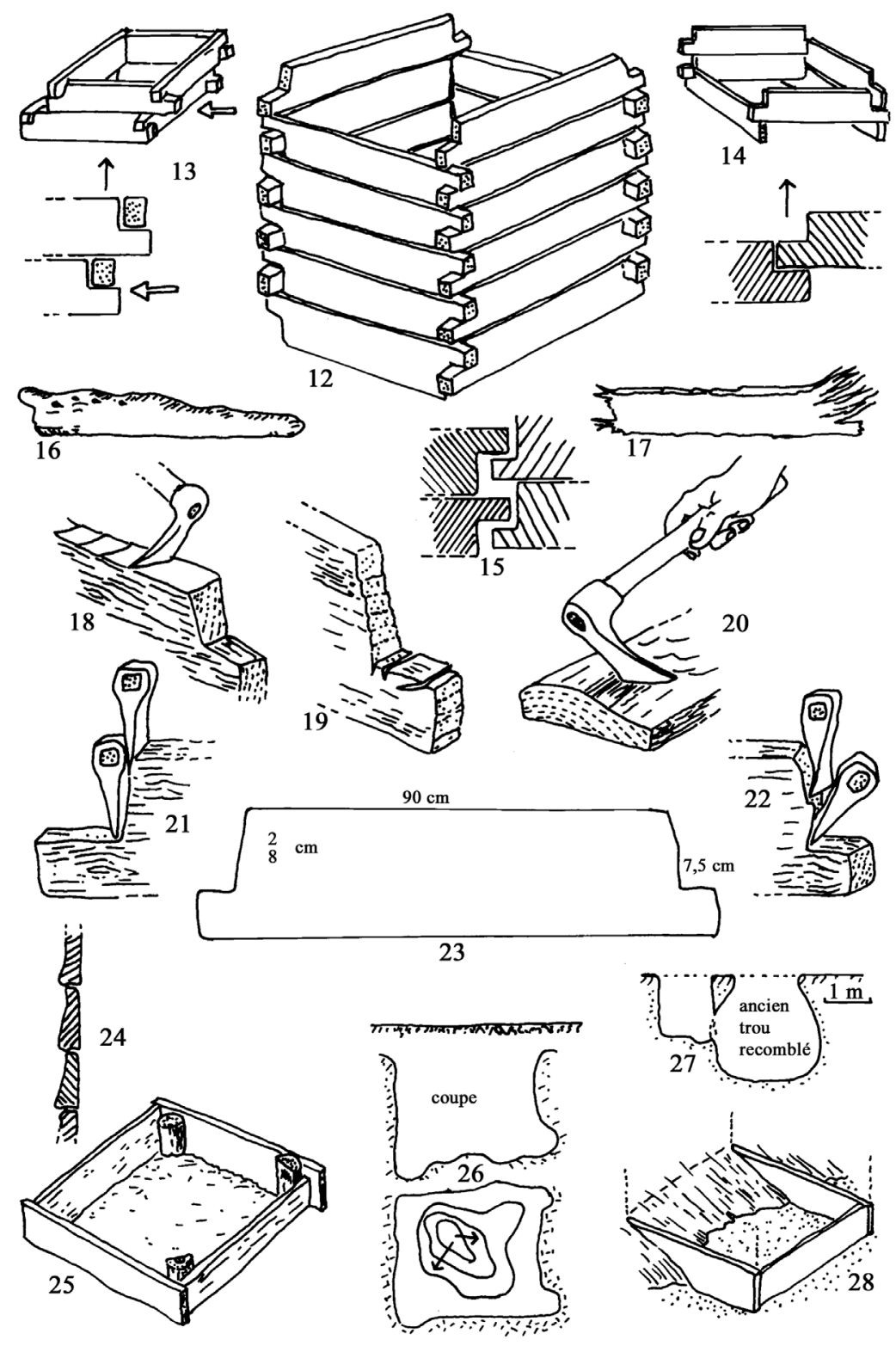

Figure $2: 12$ : reconstitution d'un cuvelage (à partir des observations effectuées sur le puits $\mathrm{n}^{\circ}$ 5) ; 13-14 : hypothèses d'assemblage à mi-bois (non retenues pour manque de stabilité ou solidité) ; 15 : hypothèse retenue d'un assemblage « au tiers ", à tenons inversés non porteurs; 16 : pièce de bois érodée à son extrémité; 17 : pièce de bois déchiquetée à ses extrémités; 18-22 : procédés de façonnage à l'herminette reconstitués d'après les traces d'outils; 23 : un bastaing type; 24 : une paroi de cuvelage en section, la surépaisseur de chaque bastaing en partie basse protégeant la jonction avec le suivant; 25 : boisage léger en planches calées par des piquets d'angle; 26-27 : coupes de deux puits abandonnés en cours de forage (cf. texte); 28 :boisage incomplet (planches encastrées dans l'argile du substrat).

Figure 2: 12: reconstruction of a timbering (from observations in well n ${ }^{\circ}$ ); 13-14: "half-wood" assembling hypotheses (not satisfactory for lack of stability or solidity); 15: retained hypothesis of "one-third" assembling, with inverted and non-bearing tenons; 16: end-eroded timber; 17: end-jagged timber; 18-22: adze-working processes reconstructed from tools traces on beams; 23: a model beam; 24: section through a timber wall: over-thickness at bottom of each beam protects its joint with the lower one; 25: lighter timbering: planks blocked by angle posts; 26-27: sections through two wells abandoned in course of digging (see in text); 28: partial timbering of planks fitted in substratum's clay. 
tement sur celui qui lui est sous-jacent. Les tenons ne sont donc plus porteurs et on a presque un tiers du jeu pour résorber les éventuelles inégalités dans la hauteur des bastaings. Cette technique est donc très intelligemment conçue $\left(n^{\circ} 15\right)$.

Par contre, faute d'outillage perfectionné, la réalisation est assez peu soignée. Les bastaings ont été obtenus par fente, selon le fil du bois, dans des troncs de chêne. Ce travail a pu être fait au coin ou à la hache frappée. Dans ces conditions le parallélisme des faces n'est qu'approximatif ( $n^{\circ} 18$ à 24); il n'y a eu ni sciage, ni rabotage. Toutefois, lorsque la distorsion des faces était trop grande, un travail à l'herminette a nivelé les bombements par des enlèvements parallèles, pour la plupart très plats (fig. $2, n^{\circ} 20$; fig. 3, $n^{\circ} 29$ ). Un coup d'herminette unique, pratiqué transversalement, je ne sais pourquoi, sur le bastaing B2 a permis d'évaluer la largeur du fer à $7,5 \mathrm{~cm}$; c'est exactement la mesure d'une herminette retrouvée dans le puits 150 de notre site (cf. infra, n 32 ).

Le travail avec ce type d'outil a parfois été effectué sur la tranche des bastaings par petits coups rapprochés espacés de 4 à $5 \mathrm{~cm}\left(\mathrm{n}^{\circ} 18\right)$. La section de nos pièces de bois étant le plus souvent en forme de trapèze allongé, c'est la plus petite base qui a été régulièrement placée vers le haut $\left(\mathrm{n}^{\circ} 24\right)$.

Le tronçonnement des extrémités et le dégagement du tenon d'assemblage, avec sectionnement par le travers du fil du bois, a été pratiqué à la hache; il est de ce fait assez grossier et ignore l'angle droit. Dans un premier cas l'extrémité présente an angle rentrant assez obtus ( $\mathrm{n}^{\circ} 21$ et 23 à gauche). La hache s'abattant pourtant bien perpendiculairement au bois ne peut, du fait de sa section triangulaire et du frottement du flanc de la lame contre la tranche ligneuse, suivre qu'une ligne oblique, et ce dès le second coup. Nous avons observé ce fait 16 fois. Dans un second cas, voulant réagir contre la tendance précitée, l'ouvrier a incliné son instrument pour donner les coups terminaux. La tranche présente alors un bombement central bien observé dans huit cas ( $n^{\circ} 22$ et 23 à droite).

Si l'on considère qu'en fin de course le fer de la hache, qui a perdu alors presque toute sa force vive, ne tranche plus mais écrase seulement une ligne de fibres de bois, on peut, en comptant les zones ainsi meurtries, estimer que cinq à huit coups de hache ont été donnés pour trancher la partie la plus haute de l'extrémité des bastaings. Les zones écrasées se marquent aussi souvent par de légers reliefs échelonnés (n 19). S’il frappe de façon répétée dans la même entaille, le fer est rapidement stoppé; aussi l'artisan, en bon bûcheron, a su alterner les coups verticaux et des coups très obliques proches de l'horizontale, pour détacher des copeaux du fond de l'encoche. Les derniers coups portés, tant verticaux qu'obliques, se lisent aisément sous forme d'incisions nettes. Sur un exemplaire j'en ai relevé 13 verticales dont deux doubles et cinq obliques. D'après les traces relevées, quatre à cinq coups ont suffi pour trancher l'extrémité des tenons. Une hache assez lourde a dû être employée; la hachette à douille trouvée dans le puits 369 (cf. infra, pl. III, n 35) aurait été incapable de travailler ainsi le chêne.

La longueur des bastaings, tenons non compris, est la seule mesure à respecter impérativement pour avoir un cuvelage à intérieur carré. En pratique, elle varie en effet assez peu allant de 87 à $93 \mathrm{~cm}$ et donnant une mesure moyenne de 90,88 $\pm 2,4 \mathrm{~cm}$. L. Goulpeau, du laboratoire d'archéométrie de Rennes a bien voulu m'indiquer que cette valeur est très proche de 3 pieds romains de $29,54 \mathrm{~cm}$ $(88,62 \mathrm{~cm})$. Par ailleurs, si l'on mesure les tenons depuis leur base jusqu'à la première incision verticale et non jusqu'à l'extrémité qui est souvent bombée, on obtient des chiffres très proches de $7,5 \mathrm{~cm}$ qui est la palme romaine $\left(n^{\circ} 23\right)$. On a vu plus haut que c'était aussi la largeur de l'herminette retrouvée sur le site ${ }^{2}$.

Les 31 puits des Pichelots ont des sections carrées avec des largeurs pouvant exactement convenir à des cuvelages de la taille de celui que nous avons retrouvé. Ces cuvelages étaient montés de haut en bas en suivant la progression du creusement. Nous en avons pour preuve deux puits amorcés et non terminés, qui présentent l'un et l'autre les rainures verticales ou les trous échelonnés destinés à recevoir les extrémités des bastaings qui dépassent. L'un a été arrêté pour une raison inconnue à $1,4 \mathrm{~m}$ de profondeur $\left(\mathrm{n}^{\circ} 26\right)$ et l'autre à $1,6 \mathrm{~m}$ parce qu'il a rencontré fortuitement une grande fosse comblée dont le souvenir avait été perdu $\left(\mathrm{n}^{\circ} 27\right)$.

Il n'est pas certain que cette technique de boisage avec de lourds bastaings de $5 \mathrm{~cm}$ d'épaisseur ait été utilisée pour tous les puits. Un fond de puits (locus 47) nous a livré, très pourri, le reste d'un encadrement fait de quatre planches de $2 \mathrm{~cm}$ d'épaisseur environ, dont une seule est bien conservée. Elles se croisent d'assez peu dans les angles du carré, mais trois poteaux, dont un seul véritablement trouvé en place, devaient être plantés dans les angles. Deux de ces poteaux appointés sont les deux moitiés d'un même tronc de $12 \mathrm{~cm}$ de diamètre, fendu par son milieu ( $\left.\mathrm{n}^{\circ} 25\right)$.

Un autre fond de puits (locus 16) est bordé, sur trois côtés seulement, de planches s'appuyant bout à bout et à angle droit dans les angles sans autre soutien $\left(n^{\circ} 28\right)$. Elles tiennent bien ce pendant, car elles ont été à moitié enfoncées dans une couche d'argile en place qui n'occupe qu'une partie $\mathrm{du}$ fond, se terminant en biseau pour laisser place à la couche de graviers sous jacents d'où sourd l'eau de la nappe. Le carré a, lui aussi, $90 \mathrm{~cm}$ de côté. Trois morceaux de planche

2. Les cernes relevés sur la tranche de ces bois par les soins du laboratoire de dendrochronologie de Besançon (G. Lambert) couvrent 154 ans. Cette séquence atlantique n'a pas pu être raccordée aux étalons suisses et allemands. 


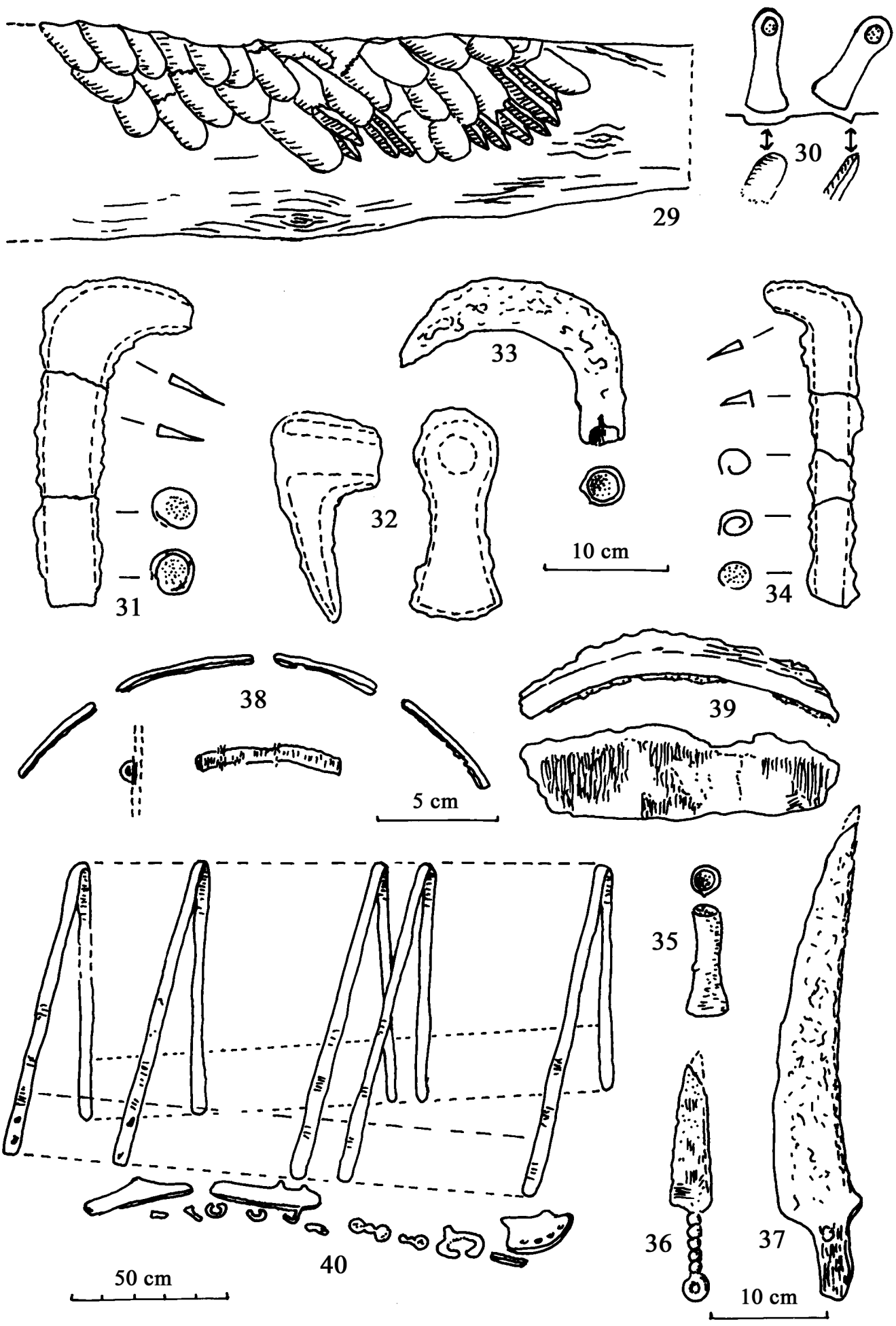

Figure 3 : 29-30 : détail des traces d'herminette relevées sur un bastaing et leur interprétation; 31-37 : outils en fer (serpette, faucille, herminette, hachette, couteaux) avec leurs traces d'emmanchement en bois; 38-39 : éléments de cerclage de récipients en bois; 40 : ferrures du locus 27 (cf. texte).

Figure 3: 29-30: detail of adze traces on a beam and their interpretation; 31-37: iron tools (billhook, sickle, adze, hatchet, knives) with traces of wooden hafting; 38-39: parts of wooden vessels hoopings; 40: iron hinges from locus 27 (see in text). 
non en place pouvaient provenir du troisième côté ou des assises d'au-dessus.

\section{TRACES DE Fibres LigneUSES CONSERvÉES PAR LA ROUILLE (fig. 3)}

Trouver des empreintes de bois dans le creux des douilles d'instruments de fer est quasi constant sur notre site et ne nous apprend rien, car on se serait évidemment douté que serpettes, faucilles, hachettes, herminettes ou même douilles indéterminées, isolées par cassure, avaient initialement des manches ou poignées de bois ( $\mathrm{n}^{\circ} 31$ à 35$)$.

- Une sorte de coutelas de boucher incurvé, garde traces d'un manche de bois riveté encadrant sa soie plate $\left(\mathrm{n}^{\circ} 37\right)$.

- C'est probablement à une gaine de bois qu'appartiennent les fibres collées par les oxydes contre la lame d'un couteau à poignée bouletée ( $\left.\mathrm{n}^{\circ} 36\right)$.

- Un demi-jonc de fer incurvé, d'une hauteur de $14 \mathrm{~mm}$ pour une épaisseur de $5 \mathrm{~mm}$ (puits 39) montre, du côté interne, une série de fibres imprimées dans la rouille qui lui sont perpendiculaires. C'est de toute évidence le cerclage d'un seau de bois, de hauteur inconnue mais d'un diamètre externe (non compris le demi-jonc) de $23 \mathrm{~cm}$ ( $\mathrm{n}^{\circ} 38$ ).

- Appartenant aussi au cerclage d'un seau probablement, puisque sa concavité est couverte de fibres transverses, un unique élément courbe qui a $3 \mathrm{~cm}$ de haut pour $2 \mathrm{~cm}$ d'épaisseur est notablement plus volumineux que le précédent, alors que sa courbure indique un récipient à peine plus grand avec $26 \mathrm{~cm}$ de diamètre (silo 213; $\mathrm{n}^{\circ} 39$ ). La réputation des tonneliers gaulois étant bien assise, ces deux trouvailles ne surprennent pas.

Sont énigmatiques, par contre, les trouvailles du locus 27. Elles sont contenues dans la plus vaste fosse du gisement, fosse qui a peut-être été creusée à leur dimension dans le sommet d'un puits abandonné. Il s'agit de bandes d'un feuillard un peu épais, $2 \mathrm{~mm}$, comparables en aspect à nos cerclages de tonneaux modernes $\left(\mathrm{n}^{\circ} 40\right)^{3}$. Ces bandes, au nombre de 5, larges de $4 \mathrm{~cm}$, étaient disposées obliquement par rapport à la verticale, parallèlement entre elles, mais à des distances variables (du sud au nord : 35, 47, 12, $43 \mathrm{~cm}$ ). Elles portent irrégulièrement des trous dont certains sont encore munis de gros rivets. L'ensemble de ces bandes est replié par-dessous selon une droite oblique sans que leur parallélisme en soit affecté. On peut en conclure que quelque chose les reliait et comme elles portent toutes, sur une face, transversalement, dans la rouille, les stries de fibres ligneuses, on est conduit à penser que ce lien était des planches.

3. Cette ressemblance n'implique aucunement l'idée d'une utilisation en tonnellerie où le cerclage métallique est d'invention récente.
On a donc affaire à une surface de plus de $2 \mathrm{~m}^{2}$, formée de planches parallèles unies par des transverses de fer rivetées. À quoi pouvait servir ce grand panneau que l'on a plié en deux pour l'introduire de force dans une fosse? Il faut noter que, $3 \mathrm{~cm}$ sous le point le plus bas de ces bandes métalliques et dans un plan horizontal, on a trouvé trois épaisses plaques de fer irrégulières ayant de 20 à $40 \mathrm{~cm}$ de plus grande longueur. L'une portait un crochet à sa face inférieure, une autre présentait un bord arqué percé de 5 trous de rivetage distants de $4 \mathrm{~cm}$. Quelques centimètres plus bas, se trouvaient d'autres ferrailles : gros anneau, crochet, maillons, tiges de section carrée. Mais tout cela faisait-il partie d'un même engin, d'usage indéterminé?

\section{Armatures de fours (fig. 4)}

À plusieurs reprises, nous avons trouvé, comme effondrée sur de petites soles de foyers circulaires englobant des tessons, des morceaux d'argile cuite armés de baguettes de bois carbonisé, ou simplement leur moulage. On peut penser qu'il s'agit des débris de voûte de petits fours ménagers comme on en utilise encore au Moyen-Orient ( $\left.n^{\circ} 41\right)$. Toutefois nous n’avons pas trouvé de baguettes incurvées, mais des éléments droits d'une dizaine de centimètres de long pour un diamètre de presque un centimètre $\left(n^{\circ} 46\right)$.

\section{LE BOIS DANS LA DÉCORATION PARIÉTALE DES DEMEURES (fig. 4)}

L'image de la hutte enfumée faite de torchis grossier, chère aux manuels périmés, doit être abandonnée, on le sait. Nous n'avons pas ici de véritable torchis fait de terre et d'herbes, mais plutôt des clayonnages revêtus d'argile. Nous retrouvons dans les morceaux durcis par la cuisson, involontaire ou préméditée, les baguettes de $1 \mathrm{~cm}$ de diamètre et perpendiculairement à elles des branchettes souvent garnies de leurs feuilles. J'ai cru reconnaître l'orme et le chêne (avec un gland) dans ces empreintes sur la glaise ( $n^{\circ} 42$ et 43 ).

Le revêtement argileux de ces claies est épais et parfois soigneusement lissé. De plus il est souvent revêtu de peinture. Celle-ci, maintes fois faite et refaite, est un lait d'argile blanche ou rose, plus rarement bleutée. Nous avons même retrouvé, jetés au dépotoir, les pots contenant encore les restes de ces badigeons.

Non seulement la couleur, mais aussi le relief contribuaient au décor des parois. Nous avons en effet des demi-colonnes engagées faites de la superposition de demi-cylindres d'argile fortement mêlée de graviers. Latéralement, ils semblent se lier aux parois et sont, comme elles, lissés et peints. Ils pré- 


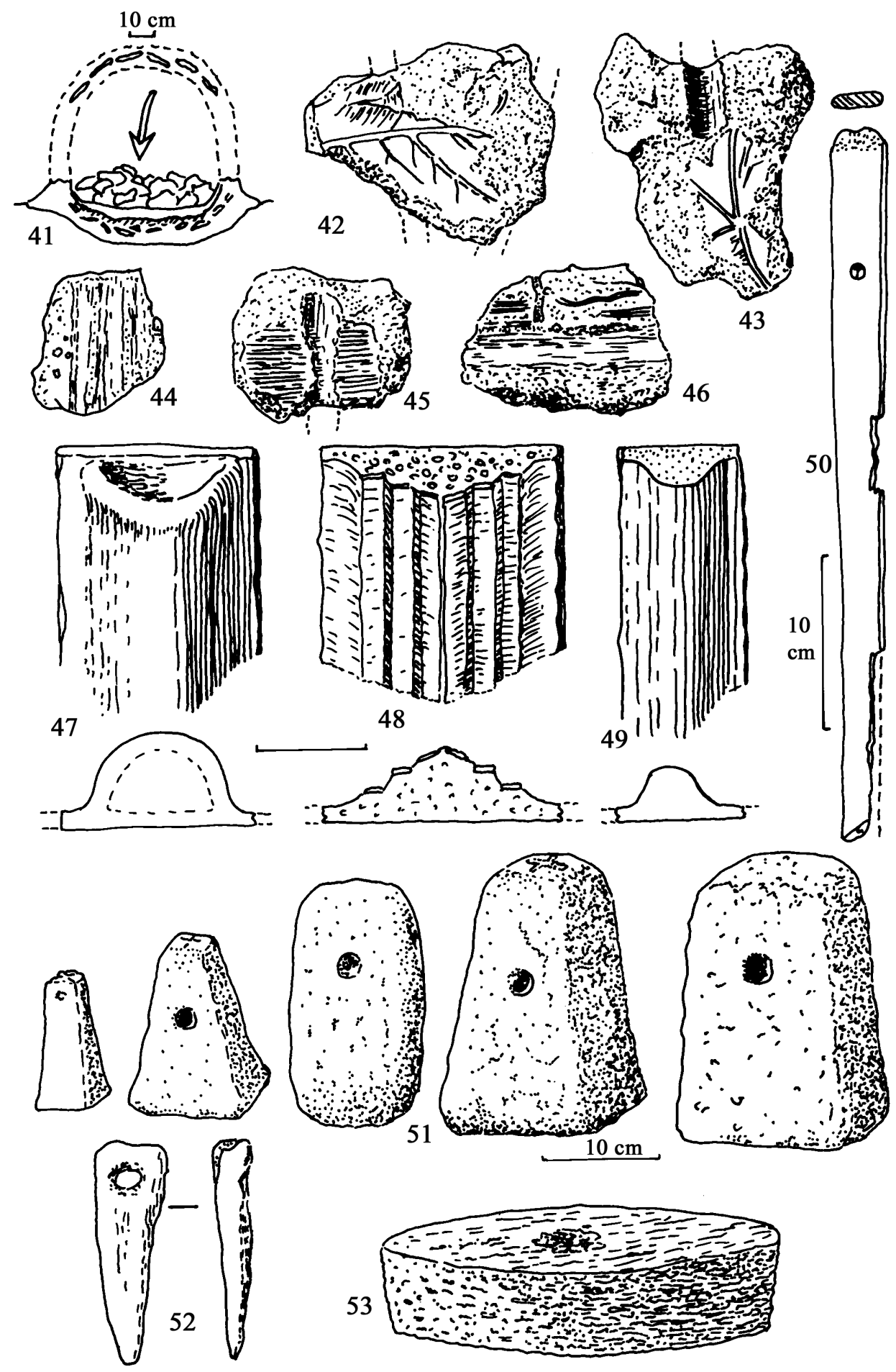

Figure $4: 41$ : sole de foyer et hypothèse de reconstitution du four domestique correspondant; 42-46 : éléments d'argile cuite armés de fragments végétaux (branchettes parfois feuillées) ; 47-49 : fragments de pilastres en torchis; 50 : possible manche en bois d'une grande louche ; 51 : série de pesons en argile; 52 : pendeloque en os; 53 : bouchon d'amphore en liège.

Figure 4: 41: hearth in section and hypothetical reconstruction of the corresponding domestic oven; 4247: pieces of baked clay armed with fragments of small branches, sometimes leafed; 47-49: fragments of cob pilasters; 50: possible wooden handle of a big ladle; 51: series of clay loom-weights; 52: bone pendant; 53: cork of an amphora. 
sentent deux diamètres, l'un de 8 , l'autre de $5 \mathrm{~cm}$ environ (n 47 et 49$)$.

Régulièrement associés à ces demi-colonnes, dans les mêmes tas de débris retrouvés dans les fosses, des reliefs argileux allongés, de section globalement triangulaire, sont armés par des baguettes dans le sens de la longueur. La face extérieure de ces reliefs est facettée en escalier par des applications de longues éclisses de bois (peut-être du châtaignier). Celles-ci sont carbonisées ou disparues, mais leur empreinte est celle de baguettes plates de $1 \mathrm{~cm}$ d'épaisseur pour une largeur de $2 \mathrm{~cm}\left(\mathrm{n}^{\circ} 48\right)$. N'ayant pas retrouvé d'angle droit affectant ces sortes de moulures, nous ne savons pas si elles constituaient des panneaux fermés. Nous les supposons verticales et parallèles aux demi-colonnes, mais sans savoir à quelle distance de celles-ci elles se trouvaient ${ }^{4}$.

\section{Autres matières OrganiQues (fig. 4)}

Le textile ne nous est connu qu'indirectement par la présence de quelques fusaïoles et de nombreux poids de métier à tisser. Une batterie de 12 très gros poids (jusqu'à $4 \mathrm{~kg}$ ) a même été trouvée rangée dans une fosse $n^{\circ} 122$ ( $\left.n^{\circ} 51\right)$.

Comme travail de l'os, bien que les ossements de cuisine soient souvent bien conservés, nous ne pouvons citer qu'un os façonné; c'est une simple pendeloque perforée, bien polie $\left(n^{\circ} 52\right)$.

Pour être complet il nous faut citer, mais comme élément importé, le bouchon de liège d'une amphore républicaine Dressel $1 \mathrm{~A}$, et la résine blonde à forte odeur vineuse qui l'englobait encore. Ce bouchon était resté dans le col, à $16 \mathrm{~cm} \mathrm{du} \mathrm{bord} \mathrm{de} \mathrm{la} \mathrm{lèvre.} \mathrm{Il} \mathrm{n'était} \mathrm{plus} \mathrm{recouvert} \mathrm{de}$ pouzzolane. Épais de $20 \mathrm{~mm}$, il est de forme tronconique aplatie avec $86 \mathrm{~mm}$ de diamètre supérieur et $81 \mathrm{~mm}$ d'inférieur. Un petit trou en son centre avait permis à la résine de trouver un chemin vers l'extérieur $\left(n^{\circ} 53\right)$. Les enduits

4. Bien distincte de ces lattes anguleuses, une tige aplatie, légèrement trapézoïdale, parfaitement polie, semble un manche cassé portant un trou de suspension. Rappelle beaucoup les manches des grandes louches de bois trouvées au site même de La Tène $\left(\mathrm{n}^{\circ} 50\right)$ intérieurs noirs de nos amphores sont, semble-t-il des goudrons minéraux.

\section{Conclusion}

En résumant les utilisations du bois aux Pichelots, on peut constater que certaines sont banales et bien connues ailleurs, ce sont les trous de poteaux, la palissade, les clayonnages, la tonnellerie, les manches d'outils. Au contraire, d'autres apparaissent plus originales, tels les cuvelages quadrangulaires des puits, les surfaces de planches unies par des feuillards rivés, les applications décoratives sur les parois.

\section{Bibliographie}

Aubin, G., Gruet, M. et Siraudeau, J., 1982 - Les monnaies gauloises des Pichelots, commune des Alleuds (Maine-et-Loire) et la chronologie des "potins ", Bulletin de la Société française de Numismatique, 37, p. 186-188.

Gruet, M. et Passini, B., 1985 - Le village ouvert des Pichelots, Tène III, Journées d'études PROLOG (Angers, 21-21 avril 1985), Nantes, Direction des Antiquités historiques des Pays de la Loire, 21 pages multigraphiées.

Gruet, M. et Passini, B., 1986 - Le village ouvert des Pichelots, Les Dossiers d'Histoire et d'Archéologie, n 106, p. 18-23.

L'Helgouac'H, J., 1998 - Michel Gruet (1912-1998) et la préhistoire en Anjou, Revue archéologique de l'Ouest, 15, p. 221-222 (suivi d'une bibliographie p. 223-227).

Santrot, J., Santrot, M.-H. et Meuret, J.-C. (dir.), 1999 - Nos ancêtres les gaulois aux marges de l'Armorique, catalogue d'exposition, Nantes, Musée Dobrée/Conseil général de LoireAtlantique, $160 \mathrm{p}$.

Siraudeau, J., 1998 - Les travaux et les jours avec le docteur Michel Gruet (1912-1998), Archives d'Anjou, n² 2, p. 5-36. 